\title{
Frequency-Reuse Planning of the Down-Link of Distributed Antenna Systems with Maximum-Ratio-Combining (MRC) Receivers
}

\author{
Eduardo Castañeda-Trujillo, Ramiro Samano-Robles and Atílio Gameiro. \\ Instituto de Telecomunicações, Campus Universitário, Aveiro, 3810-193, Portugal. \\ emails: \{ecastaneda,ramiro,atilio\}@av.it.pt
}

\begin{abstract}
Distributed antenna systems (DAS) have been shown to considerably outperform conventional cellular systems in terms of capacity improvement and interference resilience. However, the influence of frequency reuse planning on the performance of DAS remains relatively unknown. To partially fill this gap, this paper presents a comparative analysis of the down-link of DAS versus conventional cellular systems using different values of frequency reuse factor. The analysis assumes Rayleigh fading channels and it also considers maximum-ratio-combining (MRC) receivers at the user terminals to exploit diversity both in the transmission and reception links. Numerical evaluation of the analytical expressions shows that, in general, for most of the cases DAS can achieve better performance figures than conventional cellular systems using considerably smaller values of frequency reuse factor. Conversely, DAS can significantly improve the throughput $(2 x-3 x)$ and power consumption $(6-10 \mathrm{~dB})$ of conventional systems when using the same frequency reuse factor. An interesting result shows that in some particular cases DAS outperform conventional cellular systems no matter the frequency reuse factor used by the latter one, which indicates an effective capacity gain provided by the combined operation of DAS and MRC receivers.
\end{abstract}

Index Terms-Distributed antenna systems, interference resilience, frequency reuse factor, MRC receivers.

\section{INTRODUCTION}

Future wireless networks will need to provide higher data rates to larger numbers of users than current deployments. This means that increased spectral efficiency using enhanced radio resource management is required. Radio spectrum in wireless applications is an expensive resource and thus an efficient frequency reuse planning must be implemented. The frequency reuse factor $K$ defines the way in which spectral resources are allocated in a wireless deployment and it is a function of the tolerated interference in the system. The highest efficiency is achieved when in the deployment of a wireless system the smallest possible frequency reuse factor is used [1]. For example, if $K$ is reduced while capacity over a given coverage area remains constant, then less frequency resources are required and thus higher spectral efficiency is achieved.

Distributed antenna systems (DAS) were initially used to solve indoor coverage problems [2]. However, recent results indicate that DAS are also resilient to inter-cell interference [3]. In a conventional DAS deployment, antennas are geographically distributed within the cell for two main purposes: to increase coverage and to reduce the access distance to the users [4]. Each distributed antenna is connected to a central controller via a dedicated RF or optical link [5]. This architecture mimics a macroscopic multi-antenna system which can achieve low values of signal correlation [6]. Therefore, DAS will enable implementation of more efficient MIMO (multipleinput multiple-output) schemes which currently suffer from high signal correlation in co-located antenna systems (CAS).

Distributed antenna systems have been studied in previous works using several performance metrics. In [7] and [8] the authors studied the ability of DAS to save transmit power by considering different numbers of distributed antennas. Channel capacity and outage probability of DAS with fixed antenna location has been addressed in [3] and [9], while the case of random antenna location considering $K=1$ and multi-cell deployments was studied in [10]. In [6] the authors showed that DAS can provide fairness to the user population and improvements in spectral efficiency. The work in [11] studied adaptive modulation in DAS showing that significant powersavings and capacity gains over conventional systems.

Despite these recent advances in the study of DAS, the influence of frequency reuse planning on the down-link performance of DAS remains relatively unknown. Therefore, in this paper we explore the main advantages of DAS over conventional cellular systems in terms of frequency reuse factor. In addition, the analysis includes the effects of using maximum-ratio-combining (MRC) receivers at the user terminals considering different numbers of antennas that sheds light on how diversity can be exploited both in the transmission and reception links. A comparative analysis of the two deployment paradigms is here provided using outage probability and throughput as the main performance metrics. The results show that DAS can improve spectral efficiency of conventional cellular systems by increasing throughput under fixed values of frequency reuse.

Considerable improvements are further achieved when MRC is implemented at the user terminal even when the number of antennas is as low as $M=2$. Simonsson explored in [12] interference cancelation using different reuse planning techniques (Fixed and Fractional Frequency Planning) and the impact of using receivers with multiple antennas $(M=2)$ to combat the inter-cell interference for users in the cell-edge. 
The results show that the combination of MRC techniques and fractional frequency planning can improve the SINR for users located far from the base station and reduce the interference significantly. In this work we tackled the same problem by combining MRC receivers and DAS, avoiding fractional planning to consider all radio resources available for exhaustive radio resources usage. In general, DAS with MRC at the terminals allow considerable gains with respect to previous approaches in terms of frequency reuse and capacity. An interesting result indicates that in some cases DAS achieves performance levels that conventional systems cannot match no matter the frequency reuse factor used by the latter one, which indicates the large potential of DAS architectures.

The structure of this paper is as follows. Section II describes the multi-cell deployment scenario and the propagation and signal models used. Section III defines the performance metrics to be evaluated. Section IV presents the results of numerical evaluation of the analytical expressions. Finally, Section V draws the main conclusions of the paper.

\section{SYSTEM MODEL}

\section{A. Cellular Architecture}

Consider the distributed antenna system depicted in Fig. 1. Each hexagonal cell has a radius $R$ and consists of a total of $J+1$ radiating nodes: one node located at the center of the cell $(j=0)$, and $J$ distributed nodes $(j=1, \ldots, J)$ located at a fixed distance $D_{r}$ from the center of the cell and spaced at uniform angles given by $\theta_{j}=\frac{2(j-1) \pi}{J}$. A conventional cellular system with one centralized antenna can be characterized by substituting $J=0$ in all the expressions of this paper. Two transmission modes will be analyzed. In the first one, which is called blanket transmission with repetition coding, all the nodes within one cell are assumed to transmit the same information towards a single user (Fig. 2 (b)). Each node uses a transmit power level of $\frac{P}{J+1}$, where $P$ is the total transmit power of the cell. In the second one, which is called single transmission scheme, only a single antenna is selected for transmission by the criterion of minimizing propagation pathloss (Fig. 2 (c)). The selected node uses full power $P$ while the remaining nodes are deactivated, which is the optimum power allocation strategy for the proposed scheme. At the user terminal we will assume the use of $M$ antennas followed by maximum-ratio-combining in order to evaluate diversity both in the transmission and reception links. Regarding inter-cell interference calculation, different frequency reuse factors will be evaluated considering that there is only one single user transmission in any given time/frequency/code dimension [3]. In a hexagonal cellular network the available frequency reuse factors required to have a symmetric deployment are given by the formula $K=(a+b)^{2}-a b$, where $a$ and $b$ are two positive integers [1]. For simplicity, only the central cell $(i=0)$ will be used for performance metric calculation and only one tier of 6 outer cells $(i=1, \ldots, 6)$ will be considered as source of interference as in [3]. It has been verified that the differences with more than one tier are negligible. All these interfering cells are located at a reuse distance from the central cell given by $D_{c}=R \sqrt{3 K}$ [1], and spaced at uniform angles given by $\phi_{i}=\frac{(i-1) \pi}{3}$. The distance between the $j$-th node in the $i$-th cell of the network and the user of analysis $u$ in the central cell with coordinates $\left(x_{u}, y_{u}\right)$ is denoted by $d_{i, j}^{(u)}$ and is given by the following expression:

$$
d_{i, j}^{(u)}=\sqrt{\Delta_{x_{u}}(i, j)^{2}+\Delta_{y_{u}}(i, j)^{2}},
$$

where

$$
\begin{gathered}
\Delta_{x_{u}}(i, j)=\delta(i) D_{c} \cos \phi_{i}+\delta(j) D_{r} \cos \theta_{j}-x_{u}, \\
\Delta_{y_{u}}(i, j)=\delta(i) D_{c} \sin \phi_{i}+\delta(j) D_{r} \sin \theta_{j}-y_{u},
\end{gathered}
$$

and $\delta(k)$ is a binary variable which takes a value $\delta(k)=0$ when $k=0$ and $\delta(k)=1$ when $k \neq 0$.

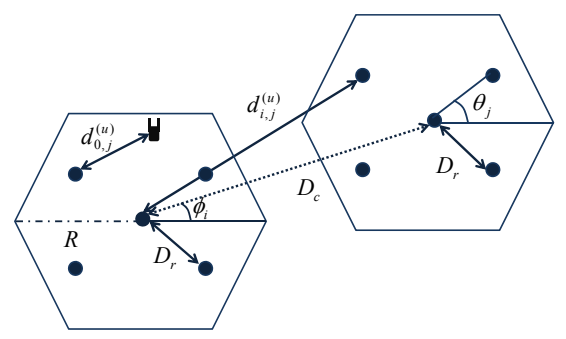

Fig. 1. Cellular Architecture for evaluation of DAS system with MRC receivers

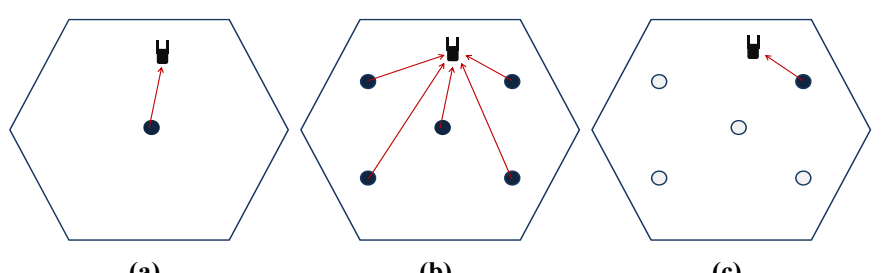

(a)

(b)

(c)

Fig. 2. Transmission Schemes used. a) is the conventional cellular system, b) is the blanket transmission scheme where all nodes transmit to the user in DAS, c) is the single transmission scheme where user is served by the best node in DAS.

\section{B. Propagation and signal models}

The channel between the $m$-th antenna of user $u$ in the central cell and the $j$-th node of the $i$-th cell of the network will be denoted by $h_{m, i, j}^{(u)}$. Channels of different users and different distributed antennas are assumed to be statistically independent, Rayleigh distributed, and affected only by a propagation path-loss model defined by $L_{i, j}^{(u)}=\left(d_{i, j}^{(u)}\right)^{-\alpha}$, where $\alpha$ is the path-loss exponent. In this paper, correlated shadowing fading has not been considered in order to simplify theoretical analysis. However, since this paper is focused on the effects of frequency reuse planning, the channel model considered is not expected to have considerable impact on the final results. The channel $h_{m, i, j}^{(u)}$ can thus be written as $h_{m, i, j}^{(u)}=\sqrt{\frac{P}{J+1} L_{i, j}^{(u)}} \psi_{m}$, for the blanket transmission scheme, or $h_{m, i, j}^{(u)}=\sqrt{P L_{i, j}^{(u)}} \psi_{m}$, for the selective transmission 
scheme, where $\psi_{m}$ is a circularly complex and zero-mean Gaussian random variable with unitary power $\psi_{m} \sim \mathcal{C N}(0,1)$.

Assuming that the signal transmitted by the $i$-th cell, which is denoted by $s_{i}$, has unitary power $\left(E\left[s_{i}^{2}\right]=1\right.$, where $E[\cdot]$ is the expectation operator) then the signal received by the $m$-th antenna of user $u$ under blanket transmission is given by:

$$
r_{u, m}=\sum_{j=0}^{J} h_{m, 0, j}^{(u)} s_{0}+\sum_{i=1}^{6} \sum_{j=0}^{J} h_{m, i, j}^{(u)} s_{i}+\nu_{u, m},
$$

while for the single transmission scheme it can be written as

$$
r_{u, m}=h_{m, 0, z}^{(u)} s_{0}+\sum_{i=1}^{6} h_{m, i, q_{i}}^{(u)} s_{i}+\nu_{u, m}
$$

where $z=\arg \max _{j \in\{0,1, \ldots, J\}}\left\{L_{0,1}^{(u)}, L_{0,2}^{(u)}, \ldots, L_{0, J}^{(u)}\right\}$ and $q_{i}$ is an integer randomly selected among $\{0,1, \ldots, J\}$ considering that the same transmission scheme is used in all the cells. This assumption is used in several papers in the literature of distributed antenna systems, e.g. [3]. The term $\nu_{u, m}$ in (1) and (2) is the noise with normalized power $\left(\nu_{u, m} \sim \mathcal{C N}(0,1)\right)$. Equations (1) and (2) can be rewritten as

$$
r_{u, m}=S_{u, m}+I_{u, m},
$$

where $S_{u, m}$ stands for all the contributions of the nodes inside the central cell, and $I_{u, m}$ represents the inter-cell interference-plus-noise term. Due to the central limit theorem, the interference-plus-noise term $I_{u, m}$ can be considered as Gaussian distributed with variance

$$
\sigma_{I_{u, m}}^{2}=\sum_{i=1}^{6} \sum_{j=0}^{J} E\left[\left|h_{m, i, j}^{(u)}\right|^{2}\right]+1
$$

for the blanket transmission scheme, and

$$
\sigma_{I_{u, m}}^{2}=\sum_{i=1}^{6} E\left[\left|h_{m, i, q}^{(u)}\right|^{2}\right]+1
$$

for the single transmission scheme. The assumption of a Gaussian distributed interference-plus-noise term has been used in other papers regarding distributed antenna systems such as [3] without affecting the final results. We have also tested this assumption by means of system level simulation and the difference can be neglected, particularly when using a large number of distributed nodes and path-loss exponents larger than 2. Since all channels are Rayleigh-distributed and the interference-plus-noise term is assumed to have Gaussian distribution (which means that the term $\sigma_{I_{u, m}}^{2}$ is seen as a constant for user $u$ and antenna $m$ ), then the signal-tointerference-plus-noise ratio at each antenna element can be proved to have exponential distribution [13]:

$$
\gamma_{u, m}=\frac{\left|S_{u, m}\right|^{2}}{\sigma_{I_{u, m}}^{2}}, \quad f_{\gamma_{u, m}}\left(\gamma_{u, m}\right)=\frac{1}{\bar{\gamma}_{u, m}} e^{-\frac{\gamma_{u, m}}{\bar{\gamma}_{u, m}}}
$$

where $\bar{\gamma}_{u, m}$ is the average SINR. Since the denominator of the SINR expression is assumed to be a constant (due to the Gaussian distribution assumption of the denominator as previously explained) then the average SINR can be calculated by applying the expectation operator to the numerator only while using in the denominator the variance of the Gaussiandistributed interference-plus-noise term. This assumption has been used in other papers dealing with the analysis of systems at the multi-cell level such as [13]. The expression of the average SINR for the blanket transmission scheme is thus given by:

$$
\begin{gathered}
\bar{\gamma}_{u, m}=\frac{E\left[\left|S_{u, m}\right|^{2}\right]}{\sigma_{I_{u, m}}^{2}}=\frac{E\left[\left|\sum_{j=0}^{J} h_{m, 0, j}^{(u)}\right|^{2}\right]}{\sum_{i=1}^{6} \sum_{j=0}^{J} E\left[\left|h_{m, i, j}^{(u)}\right|\right]^{2}+1} \\
=\frac{\sum_{j=0}^{J} \frac{P}{J+1}\left(d_{0, j}^{(u)}\right)^{-\alpha}}{\sum_{i=1}^{6} \sum_{j=0}^{J} \frac{P}{J+1}\left(d_{i, j}^{(u)}\right)^{-\alpha}+1}
\end{gathered}
$$

and for the single transmission scheme by:

$$
\bar{\gamma}_{u, m}=\frac{\left(d_{0, z}^{(u)}\right)^{-\alpha}}{\sum_{i=1}^{6}\left(d_{i, q}^{(u)}\right)^{-\alpha}+\frac{1}{P}}
$$

Since the previous expressions are independent of $m$ then the term $\bar{\gamma}_{u, m}$ will be simply denoted by $\bar{\gamma}_{u}$.

\section{PERformanCE METRICS}

\section{A. Outage probability}

The outage probability is a metric used to evaluate system coverage performance and it is defined as the probability that the instantaneous SINR fails to achieve a target SINR value. In this paper, the outage probability of a user $u$ in a particular location in the central cell is defined as the probability that the instantaneous SINR $\gamma_{u}$ lies below a target SINR $\gamma_{T}$. This can be written in mathematical form as:

$$
P_{\text {out }}^{(u)}=\operatorname{Pr}\left\{\gamma_{u} \leq \gamma_{T}\right\},
$$

which is equivalent to the cumulative density function of the SINR of user $u$ : $F_{\gamma_{u}}\left(\gamma_{T}\right)$. Using the signal model in the previous section, this outage probability can be calculated in closed-form for a single antenna receiver using the Cumulative Distribution Function (CDF) of the exponential distribution distribution [14]:

$$
P_{\text {out }}^{(u)}=1-e^{-\frac{\gamma_{T}}{\bar{\gamma}_{u}}}
$$

However, in this paper we assume an MRC receiver whose main characteristic is that its output SINR is the summation of the SINRs of the individual antennas $\left(\gamma_{u}=\sum_{m} \gamma_{u, m}\right)$. In a scenario without correlation this means that $M$ exponentially identical and independently distributed random variables are added. The result is a central Chi-square distribution with $2 M$ degrees of freedom with CDF given by the closed-form expression [14]:

$$
P_{\text {out }}^{(u)}=1-e^{-\frac{\gamma_{T}}{\bar{\gamma}_{u}}} \sum_{k=0}^{M-1} \frac{1}{k !}\left(\frac{\gamma_{T}}{\bar{\gamma}_{u}}\right)^{k}
$$

Now it is possible to calculate the average outage probability across the cell by integrating the previous expression across 
the set of all user positions in the cell, denoted by $\mathcal{U}_{0}$, as follows:

$$
P_{\text {out }}=\int_{u \in \mathcal{U}_{0}} P_{\text {out }}^{(u)} \operatorname{Pr}\{u\} d u,
$$

where $\operatorname{Pr}\{u\}$ is the probability of occurrence of the user location $u$. We will now assume uniform user distribution across the cell, which means all user positions are equally likely and which allows us to numerically evaluate the above integral as a simple averaging operation:

$$
P_{\text {out }}=\frac{1}{N_{u}} \sum_{u=1}^{N_{u}} P_{\text {out }}^{(u)},
$$

where $N_{u}$ is the number of user positions considered in the numerical evaluation.

\section{B. Throughput}

The throughput of a system can be defined as the long term ratio of the total number of bits correctly received by the users to the total time spent to transmit such information. The throughput is thus a metric that measures the service quality in a system. In systems with fixed transmission interval the throughput can be expressed in mathematical form as the ratio of average number of bits correctly transmitted per TTI (transmission time interval) or $E\left[I_{b}\right]$ to the duration of the TTI, denoted by $L$ :

$$
T=\frac{E\left[I_{b}\right]}{L}
$$

Since the transmission of information bits in a real network commonly takes place in blocks or packets, the average number of correctly transmitted bits per TTI can be expressed as the product of the correct packet or block reception probability times the number of bits transported by a packet $B$ :

$$
E\left[I_{b}\right]=B \operatorname{Pr}\{\text { correct packet reception }\},
$$

The correct packet reception probability can be also expressed as:

$$
\operatorname{Pr}\{\text { correct packet reception }\}=\operatorname{Pr}\{t=1\},
$$

where $t$ is a binary random variable with value 0 when the packet is incorrectly received and $t=1$ when it is correctly received. The conditional probability of correct packet reception at a particular user location $u$ can be approximated by the probability of the SINR of user $u$ to surpass a given threshold $\gamma_{T_{m c s}}$ of the particular modulation and coding scheme being used:

$$
\operatorname{Pr}\{t=1 \mid u\}=\operatorname{Pr}\left\{\gamma_{u}>\gamma_{T_{m c s}}\right\},
$$

This SINR reception model considers that all bits in a packet have been correctly received if the SINR surpasses the threshold $\gamma_{T_{m c s}}$, which is an optimistic assumption. However, at the system level we have confirmed that this SINR packet reception model provides a very good approximation to the real system performance using look-up tables. Furthermore, this assumption has been extensively used in literature, e.g. [15][18], and it is used here to facilitate analytical work without sacrificing the accuracy of the final results. The thresholds for real systems can be obtained from tables of modulation and
TABLE I

WIMAX MODULATION AND CODING SCHEMES

\begin{tabular}{|c||c||c||c||c|}
\hline MCS level & BLER & AMC (R) & $\gamma_{T} \mathrm{~dB}$ & Bits (B) \\
\hline 1 & $4.10 \mathrm{e}-3$ & QPSK - 1/3 & -1.14 & 2 \\
\hline 2 & $4.12 \mathrm{e}-3$ & QPSK - 1/2 & 1.32 & 2 \\
\hline 3 & $7.15 \mathrm{e}-3$ & 16QAM - 1/3 & 6.52 & 4 \\
\hline 4 & $3.30 \mathrm{e}-3$ & 16QAM - 4/5 & 11.67 & 4 \\
\hline
\end{tabular}

coding schemes of conventional wireless networks. Without loss of generality, we use LUTs of WiMAX to evaluate the system considering that the theoretical analysis is applicable for any other wireless technology. Table I shows an example of the modulation and coding schemes (MCS) used in WiMAX networks [19]. The MCS level 0 corresponds to a SINR lower than the minimum threshold, therefore no service is provided.

The global throughput for fixed modulation and coding schemes can thus be expressed as:

$$
T=\int_{u \in \mathcal{U}_{0}} \frac{B_{s} R_{c} N_{p}}{L}\left(1-F_{\gamma_{u}}\left(\gamma_{T}\right)\right) \operatorname{Pr}\{u\} d u
$$

where $B_{s}$ is the number of bits per symbol, $R_{c}$ is the coding rate and $N_{p}$ is the number of symbols per block. The extension to systems with adaptive modulation and coding is straightforward:

$$
\begin{gathered}
T=\int_{u \in \mathcal{U}_{0}} \sum_{m c s=0}^{N_{m c s}} \frac{B_{s}^{(m c s)} R_{c}^{(m c s)} N_{p}}{L} \\
\times\left(F_{\gamma_{u}}\left(\gamma_{T_{m c s+1}}\right)-F_{\gamma_{u}}\left(\gamma_{T_{m c s}}\right)\right) \operatorname{Pr}\{u\} d u,
\end{gathered}
$$

where $N_{m c s}$ is the number of modulation and coding schemes.

\section{Results}

This section presents the results of the numerical evaluation of the analytical expressions presented in the previous section. The results were obtained by using ten thousand user positions within the cell of analysis. Our approach is similar to a system level simulator, but the performance of the physical layer is absorbed by making a numerical evaluation of the expressions derived in previous subsections under rayleigh fading channels rather than generating the actual channel instances. The distributed nodes are located at a distance $D_{r}=\frac{2}{3} R$ in order to have a uniform distribution of the nodes across the all system deployment area. The number of radiating nodes used is $J=4$ for DAS and a path-loss exponent $\alpha=4$. Throughput evaluation was performed assuming the following transmission parameters of a WiMAX system: one single block of $N_{p}=7200$ symbols is transmitted to one single user per frame or TTI with a frame duration of $L=5(\mathrm{~ms})$.

The total transmit power per cell $P$ to be used in the evaluation of all the expressions derived in this paper, particularly (3) and (4), can be obtained by defining a reference average SINR value $\left(S I N R_{r e f}\right)$ that must be achieved at the edge of the cell of a conventional system without considering interference:

$$
\frac{P}{\sigma_{I_{u_{r e f}}}^{2}}=\frac{10^{S I N R_{\text {ref }} / 10}}{R^{-\alpha}},
$$


where $\sigma_{I_{u_{r e f}}}^{2}$ is the variance of the interference-plus-noise term of a reference user located at the edge of the cell and which due to the absence of interference in the reference scenario it has a unitary value $\left(\sigma_{I_{u_{r e f}}}^{2}=1\right)$.

\section{A. Outage Probability}

Fig. 3 displays the average outage probability across the cell $P_{\text {out }}$ from (5) versus the reference SINR $\left(S I N R_{\text {ref }}\right)$ from (7) for conventional cellular and DAS systems with one antenna at the MRC receiver $(M=1)$. The results assume a threshold $\gamma_{T}$ for 16-QAM modulation, which corresponds to MCS level 4 in Table I. The two DAS transmission modes are denoted by $D A S_{b}$ for blanket transmission and $D A S_{s}$ for single transmission scheme. It can be observed that for values of $S I N R_{\text {ref }}<9 \mathrm{~dB}, D A S_{s}$ with frequency reuse factor $K=1$ shows a better outage probability than conventional systems with $K=7$. This suggests that in terms of outage probability $D A S_{s}$ can improve spectrum usage by reducing the required reuse factor to provide a given service. Consider now a fixed value of $P_{\text {out }}=10^{-1}$ in Fig. 3. To achieve this condition $D A S_{s}$ with $K=3$ requires $S I N R_{\text {ref }}=5 \mathrm{~dB}$, whereas $D A S_{b}$ with $K=3$ requires $S I N R_{\text {ref }}=20 \mathrm{~dB}$. In the case of conventional cellular systems only the system with $K=7$ achieves this probability with $S I N R_{r e f}=18 \mathrm{~dB}$ while all other cases never reach the target $P_{\text {out }}$ value. In the particular case of $D A S_{s}$ with $K=7$ it can be observed that DAS can reach performance levels that conventional architectures will never reach, no matter the value of frequency reuse factor used. This result demonstrates the effectiveness of DAS to improve spectral efficiency of cellular systems.

Fig. 4 shows similar results to the ones in Fig. 3 but this time considering an MRC receiver with two antennas $(M=2)$. In this case, $D A S_{s}$ system with $K=1$ presents a better outage probability than conventional systems for values of $S I N R_{\text {ref }}<6 \mathrm{~dB}$, even if the frequency reuse factor $K$ of the latter one is as high as 21 . The improvement in outage probability by implementing MRC at the receiver is more significant for large values of $S I N R_{r e f}$.

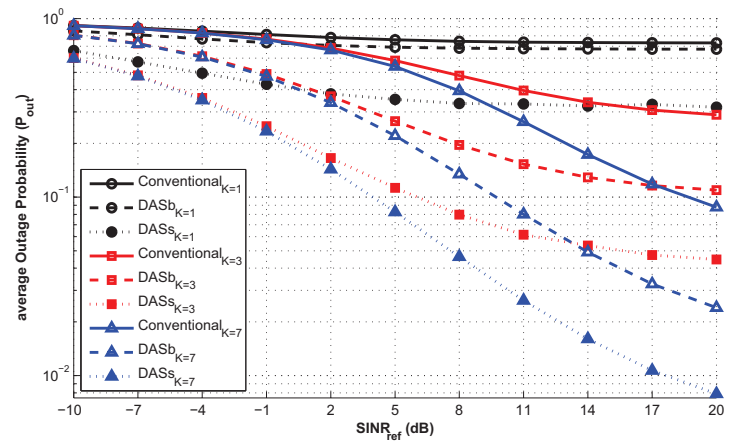

Fig. 3. Outage Probability $P_{\text {out }}$ vs. SINR $R_{\text {ref }}$ of conventional cellular and distributed systems (blanket $D A S_{b}$ and single antenna transmission $D A S_{s}$ ) considering a single antenna receiver and different values of $K$.

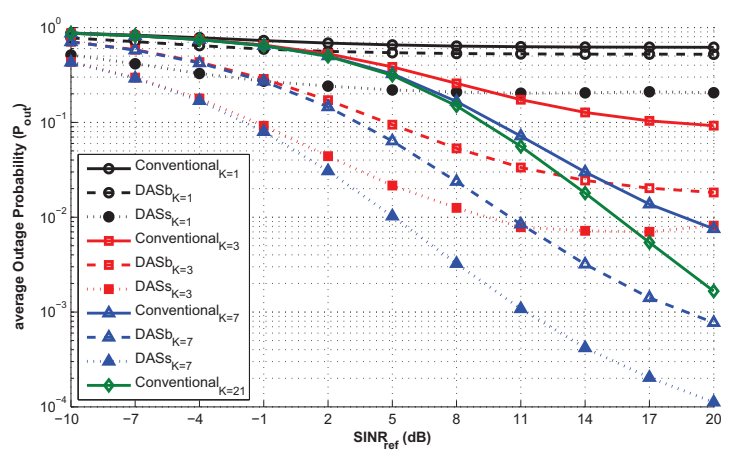

Fig. 4. Outage Probability $P_{\text {out }}$ vs. SINR $R_{\text {ref }}$ of conventional cellular and distributed systems (blanket $D A S_{b}$ and single antenna transmission $D A S_{s}$ ) considering an MRC receiver $M=2$ with uncorrelated channels and different values of $K$.

\section{B. Cell Throughput}

Fig. 5 presents the average throughput $T$ in (6) versus the reference SINR $\left(S I N R_{r e f}\right)$ in (7) using different values of frequency reuse factor $K$ and considering an MRC receiver with two antennas $(M=2$.) In order to achieve a target average throughput of $4(\mathrm{Mb} / \mathrm{s})$ and considering a fixed frequency reuse factor $K=3, D A S_{s}$ and $D A S_{b}$ require a value of $S I N R_{\text {ref }}$ of $-8 \mathrm{~dB}$ and $-2 \mathrm{~dB}$, respectively. By contrast, conventional cellular systems require more than 5 $\mathrm{dB}$. Therefore, DAS can achieve the same performance as conventional systems using lower values of transmit power. For a fixed SINR reference value of $S I N R_{\text {ref }}=-1 \mathrm{~dB}$ and $K=3$ the average throughput achieved by $D A S_{s}$ and $D A S_{b}$ surpasses the average throughput of conventional cellular system by $150 \%$ and $137 \%$ respectively.

Fig. 6 shows the cumulative distribution function of the user throughput assuming $S I N R_{\text {ref }}=0 \mathrm{~dB}$ and two antennas at the MRC receiver $(M=2$.) If the minimum required throughput is $2 \mathrm{Mbps}$ or higher, $D A S_{s}$ with $K=1$ achieves higher throughput than conventional systems with $K=3$ and $K=7$. If the frequency reuse is fixed in both systems $K=3$, the minimum average throughput that a user can achieve in $D A S_{s}$ and $D A S_{b}$ systems with two antennas at the MRC receiver is $2.9(\mathrm{Mb} / \mathrm{s})$ and $2.4(\mathrm{Mb} / \mathrm{s})$ whereas conventional cellular system presents only $1.3(\mathrm{Mb} / \mathrm{s})$.

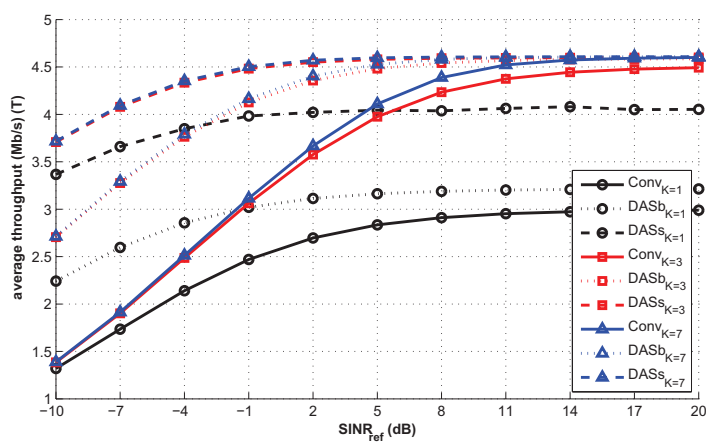

Fig. 5. Average throughput vs. $S I N R_{r e f}$ of conventional and distributed antenna systems (blanket $D A S_{b}$ and single $D A S_{s}$ transmission schemes) using different values of $K$ with two antennas $(M=2)$ at the MRC receiver. 


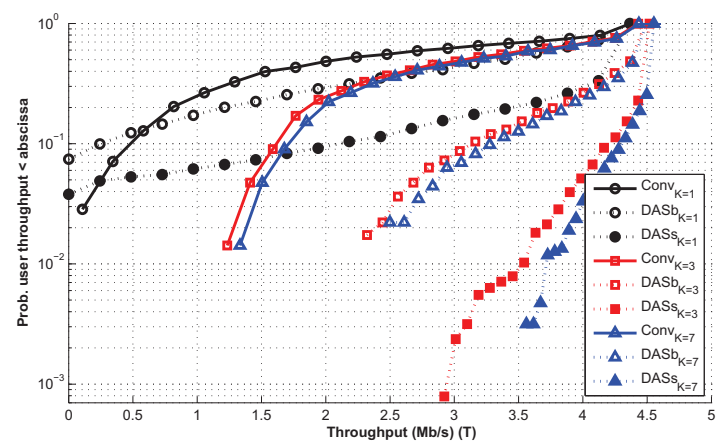

Fig. 6. Throughput CDF of conventional cellular and distributed antenna systems (blanket $D A S_{b}$ and single $D A S_{s}$ transmission schemes) for different $K$ and two antennas at the MRC receiver $(M=2)$.

Fig. 7 shows the average throughput that can be achieved when MRC receiver is implemented with different number of antennas. The numerical evaluation considered more than 4 antennas at the receiver, this scenario is not realistic but it helps to show the asymptotical behavior of the achievable throughput as the number of antennas at the receiver increases. The average throughput achieved by $D A S_{b}$ for $K=1$ with one and two antennas at the MRC receiver is similar to the one achieved by conventional system with $K=3 . D A S_{s}$ scheme shows the best average throughput specially when the number of antennas at the MRC receiver is less than 4 .

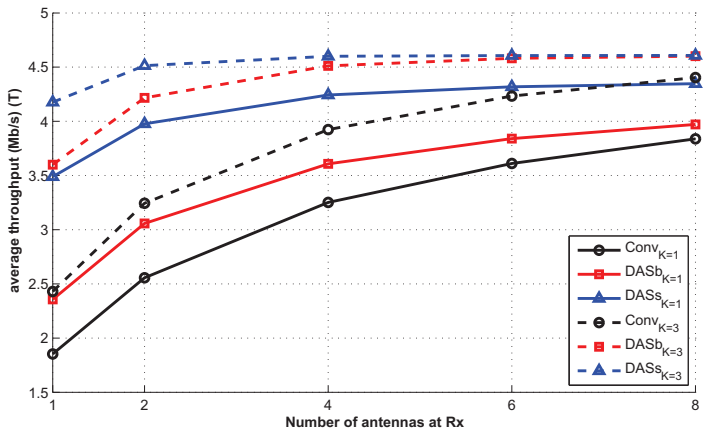

Fig. 7. Average throughput vs. Number of antennas at the receiver (MRC) of conventional and distributed antenna systems (blanket $D A S_{b}$ and single $D A S_{s}$ transmission schemes) assuming $S I N R_{r e f}=0 \mathrm{~dB}$ and $K=1$ and $K=3$.

\section{CONCLUSIONS}

In this paper we have analyzed the advantages of using distributed antenna systems over conventional cellular systems in terms of the frequency reuse planning, outage probability and average throughput. In addition, both systems have been enhanced by using MRC receivers at the mobile terminals with various numbers of antennas. The results of this paper show that the distributed antenna architectures can be used to increase the average throughput with respect to a conventional cellular deployment by more than $180 \%$ and $220 \%$ for blanket $\left(D A S_{b}\right)$ and single transmission scheme $\left(D A S_{s}\right)$, respectively. $D A S_{s}$ has shown to be the best transmission scheme, it allows us to improve the metrics $P_{\text {out }}$ and $T$ compared to conventional system with less power and small values of $K$. The average throughput can be further improved by implementing MRC at the receiver and the results show that this is particularly useful for low values of transmit power. The distributed antennas architecture can reduce the frequency reuse thus maximizing the spectrum efficiency and increasing the coverage area or cell size with the same power budget. It was also found that in some particular cases DAS outperform conventional cellular systems no matter the frequency reuse factor used by the latter one, which indicates that DAS provides an effective increase of spectral efficiency.

\section{ACKNOWLEDGMENTS}

The authors would like to thank the valuable suggestions from anonymous reviewers. The work was partially supported by the Seventh Framework Program for Research of the European Commission, under FET-Open grant CROWN (PTDC/EEA-TEL/115828/2009).

\section{REFERENCES}

[1] Rappaport T. Wireless communications: principles and practice, Prentice Hall, 2002.

[2] Saleh A. M., Rustako A., and Roman R., "Distributed antennas for indoor radio communications," IEEE Transactions on Communications, vol. 35, pp. 1245-1251, Dec. 1987.

[3] Wan Choi, Andrews J.G. and Chaehag Yi, "Capacity of multicellular distributed antenna networks," International Conference on Wireless Networks, vol.2, pp. 1337-1342, Dec. 2005.

[4] Zhou S., Zhao M., Xu X., Wang J. and Yao Y., "Distributed wireless communication system: a new architecture for future public wireless access," IEEE Communications Magazine, vol. 41, Mar. 2003.

[5] Wake D., Webster M., Wimpenny G., Beacham K. and Crawford L., "Radio over fiber for mobile communications," IEEE International Topical Meeting on Microwave Photonics, pp. 157-160, Oct. 2004.

[6] Toufik I. and Knopp, R., "Wideband Channel Allocation in Distributed Antenna Systems," IEEE Vehicular Technology Conference, pp. 1-5, Sept. 2006.

[7] Obaid A. and Yanikomeroglu H., "Reverse-link power control in CDMA distributed antenna systems," IEEE Wireless Communications and Networkinig Coisf, vol.2, pp. 608-612, Sept. 2000.

[8] Kudoh E. and Adachi F., "Power and frequency efficient virtual cellular network," IEEE Vehicular Technology Conference, vol.4, pp. 2485-2489, Jul. 2003.

[9] L. Dai, S. Zhou, and Y. Yao, "Capacity with MRC-based macrodiversity in CDMA distributed antenna systems," IEEE Globecoin, vol.1, pp. 987991, Nov. 2002.

[10] Zhang J. and Andrews J., "Distributed Antenna Systems with Randomness," IEEE Wireless Communications, vol. 7, pp. 3636-3646, Sep. 2008.

[11] Clark M. V. and et al., "Distributed versus centralized antenna arrays in broadband wireless networks," IEEE Vehicular Technology Conference, vol.1, pp. 33-37, May 2001.

[12] Simonsson, A., "Frequency Reuse and Intercell Interference CoOrdination In E-UTRA," Vehicular Technology Conference, pp. 3091 -3095, April 2007.

[13] Czylwik A. and Matsumoto T., "Downlink beamforming for frequencyduplex systems in frequency-selective fading," IEEE Vehicular Technology Conference, vol.2, pp. 695-699, May 2000.

[14] J. Proakis, Digital Communications, McGraw-Hill, 4th Edition, 2001.

[15] Naware V., Mergen G., and Tong L. "Stability and delay of finiteuser slotted ALOHA with multipacket reception," IEEE Transactions on Information Theory, vol.51, no.7, pp. 2636-2656, July 2005.

[16] European ICT FUTON Project. http://www.ict-futon.eu/.

[17] Farooq Khan, LTE for $4 G$ Mobile Broadband, Cambridge University Press 2009

[18] Baccelli F., Blaszczyszyn B., and Muhlethaler P. "An Aloha protocol for multihop mobile wireless networks," IEEE Transactions on Information Theory, vol.52, no.2, pp. 421-436, Feb. 2006

[19] WiMAX system level evaluation methodology, WiMAX Forum, V.0.0.1, 2006. 